\title{
Anesthetic management of a neonate with congenital laryngeal cyst
}

\author{
Yong Woo Choi, Jin Young Chon, Ho Sik Moon, Ji Yoon Kim, and Ji Young Lee \\ Department of Anesthesiology and Pain Medicine, Yeouido St. Mary Hospital, The Catholic University of Korea College of Medicine, \\ Seoul, Korea
}

Congenital laryngeal cysts are rare, with an incidence of 1.82 per 100,000 live births [1]. They arise from the glottic area $(58.2 \%)$, ventricular fold (18.3\%), vallecula (10.5\%), epiglottis $(10.1 \%)$, and the aryepiglottic fold, as an order of frequency [2]. A congenital laryngeal cyst may easily obstruct the smaller airway of a neonate [3]. During anesthesia for patients with laryngeal cysts, anesthesiolosists can face the risk of obscured views of the larynx, loss of the airway, risk of rupturing the cyst, and potential aspiration of cyst contents.

A $3.83 \mathrm{~kg}$-weighed, 21-day-old female neonate was scheduled for an elective operation on a laryngeal cyst. She was born at 39 weeks and two days of gestation by cesarean section at $3.7 \mathrm{~kg}$ body weight. She started to cough at 7 days after birth, exacerbated by feeding. Her chest x-ray was normal. The otolaryngologist heard stridor, and confirmed a laryngeal cyst by flexible fiberoptic laryngoscopy. They confirmed a $1.2 \times 0.9 \mathrm{~cm}$ - sized large fluid attenuation mass at the right oropharyngeal region immediately below the vallecula by CT (Fig. 1). Her weight gain was only 130 gm during the 3 weeks after birth. She was inactive and cried very weakly.

The major concern in anesthesia was how to intubate the trachea of the neonate. Our flexible endoscope has an outer diameter of $3.1 \mathrm{~mm}$, unusable through $3.0 \mathrm{~mm}$ and $3.5 \mathrm{~mm}$ inner diameter endotracheal tubes. We decided to attempt an awake intubation.

When the neonate arrived at the operating suite, her blood pressure was 75/40 $\mathrm{mmHg}$, heart rate was 135 beats/min, and $\mathrm{SpO}_{2}$ was $97 \%$. We injected glycopyrrolate $0.04 \mathrm{mg}$ intravenously.
After sufficient preoxygenation at right-side down decubitus position, we attempted a direct laryngoscopy with a Macintosh \#1 blade. However, while the attempted intubation failed, her $\mathrm{SpO}_{2}$ did not decrease. From a brief observation during the first attempt, the laryngeal cyst completely blocked our view of the larynx. It appeared that she did not need sedative or anesthetics for further intubation procedure because she was inactive and her struggling was very weak during laryngoscopy. After the second trial of laryngoscopy that brought the same result, we

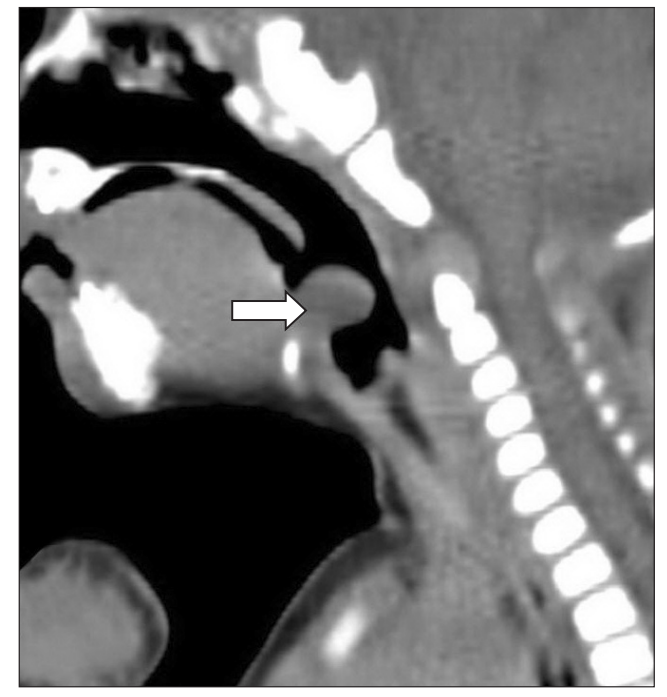

Fig. 1. Neck CT sagittal view shows a $1.2 \times 0.9 \mathrm{~cm}$ - sized cyst (arrow) just below the vallecula, protruding into the airway.

Corresponding author: Ji Young Lee, M.D., Department of Anesthesiology and Pain Medicine, Yeouido St. Mary Hospital, The Catholic University of Korea College of Medicine, 62, Yeouido-dong, Yeongdeungpo-gu, Seoul 150-713, Korea. Tel: 82-2-3779-1944, Fax: 82-2-783-0368, E-mail: leeji@catholic.ac.kr

(c) This is an open-access article distributed under the terms of the Creative Commons Attribution Non-Commercial License (http:// creativecommons.org/licenses/by-nc/3.0/), which permits unrestricted non-commercial use, distribution, and reproduction in any medium, provided the original work is properly cited. 
decided to aspirate the cystic contents . We punctured the cyst with a 22 gauge needle and aspirated about $1 \mathrm{ml}$ of the viscous fluid under the laryngoscopy at decubitus position, while an assistant suctioned the leaking fluid simultaneously. The cyst was decompressed and we intubated the trachea with a $3.0 \mathrm{~mm}$ inner diameter uncuffed endotracheal tube under the view of the entire glottis while the patient breathed spontaneously. We used $2 \%$ sevoflurane- $\mathrm{O}_{2} 1 \mathrm{~L} / \mathrm{min}-\mathrm{N}_{2} \mathrm{O} 1 \mathrm{~L} / \mathrm{min}$ through a semiclosed circuit and injected $0.5 \mathrm{mg}$ of vecuronium bromide. Excision of the cyst under a microscope by the otolaryngologist took 40 minutes. The neonate recovered spontaneous ventilation and her trachea was extubated after an injection of glycopyrrolate $0.04 \mathrm{mg}$ and pyridostigmine $1 \mathrm{mg}$. Her recovery process was unremarkable. At the follow-up to 4 months after surgery, her body weight had increased to $7.7 \mathrm{~kg}$, and stridor no longer occurred during feeding.

The presenting symptoms of laryngeal cysts can vary with age of the patient, and the size and location of the cyst. Laryngeal cysts in adults can either be asymptomatic until it is detected incidentally such as during intubation, or symptoms are similar to other space occupying lesions of the airway. In neonates and infants, they produce clinical presentations of upper airway obstruction, such as dyspnea, inspiratory stridor, and feeding difficulty soon after birth or during the first weeks of life [1-3]. In the series of pediatric vallecular cysts, the most common symptoms were stridor and feeding problems [3], causing our patient to fail to thrive. These conditions of the patient and the degree of airway obstruction influenced our plan to give her only minimal drugs during anesthesia. In diagnosis of a laryngeal cyst, flexible fiberoptic laryngoscopy is essential, as well as CT, MRI, or ultrasonography [4].

When we planned anesthesia, airway management was the most problematic aspect. There was the possibility of airway loss by sedation and paralysis. Through the first trial of direct laryngoscopy at awaken state, we judged that the neonate was tolerable for laryngoscopy and the airway was very difficult to intubate. Several reports have described the method of intubation in pediatric patients with laryngeal cysts. Ahrens et al. [4] intubated bronchoscopically through the laryngeal mask airway (LMA) in a 3-month-old infant with a vallecular cyst, using a $2.2 \mathrm{~mm}$ diameter very thin bronchoscope. In pediatric airway emergencies on children with vallecular cysts, emergency cyst puncture or tracheotomy have been performed [3]. The surgeons should be asked to be on standby for emergency tracheotomy.

We considered which laryngoscopy blade would be useful. Any blade could compress the cyst, increasing the risk of rupture and aspiration. However, Kalra et al. [5] reported successful intubation in a neonate using a right paraglossal straight blade laryngoscopy (Miller size 0 blade) with the aid of an intubating stylet. It was considered appropriate to try the paraglossal approach in our case.

The success of puncture and aspiration depends on the characteristics of the cystic contents. Very thick cystic content cannot be aspirated. For this reason, the authors recommended the use of a wide-bore needle to aspirate [1]. Fortunately in our case, the cystic contents were slightly turbid but aspirated well through a $22 \mathrm{G}$ needle. We kept in mind the possibility of pulmonary aspiration and suctioned the leaking contents at immediately below the decubitus position under laryngoscopic view.

Congenital laryngeal cysts in pediatric patients are challenging cases for anesthesiologists. When we planned anesthesia, we thoroughly evaluated the general condition of the patient and the endoscopic and radiologic results of the epiglottic cyst. We could intubate successfully after aspiration of the cyst contents while the neonate breathed spontaneously. Also, alternative methods could be to use fiberoptic intubation with an ultrathin bronchoscope, intubating LMA, or paraglossal straight blade laryngoscopic technique. Whatever intubation methods are chosen, anesthesiologists should always be reminded of the potentially life-threatening nature of congenital laryngeal cysts.

\section{References}

1. Pak MW, Woo JK, van Hasselt CA. Congenital laryngeal cysts: current approach to management. J Laryngol Otol 1996; 110: 854-6.

2. Arens C, Glanz H, Kleinsasser O. Clinical and morphological aspects of laryngeal cysts. Eur Arch Otorhinolaryngol 1997; 254: 430-6.

3. Gutiérrez JP, Berkowitz RG, Robertson CF. Vallecular cysts in newborns and young infants. Pediatr Pulmonol 1999; 27: 282-5.

4. Ahrens B, Lammert I, Schmitt M, Wahn U, Paul K, Niggemann B. Life-threatening vallecular cyst in a 3-month-old infant: case report and literature review. Clin Pediatr (Phila) 2004; 43: 287-90.

5. Kalra S, Saraswat N, Kaur R, Agarwal R. Vallecular cyst in a newborn: a challenging airway. Anaesth Intensive Care 2011; 39: 509-10. 